\title{
Relationship between the degree of dependence to nicotine, and the anxio-depressive levels, according to Fagerstrom test of nicotine dependence and HADS test
}

\author{
Aziez Chettoum, Hacène Frih", Redha Djenidi, Bachir Ali Rachedi, Abdelkrim Tahraoui
}

Laboratory of Applied Neuro-Endocrinology, Badji Mokhtar University, Annaba, Algeria

Email: ${ }^{*}$ frihhacene@yahoo.fr

Received 21 April 2012; revised 24 May 2012; accepted 8 June 2012

\begin{abstract}
The main objective of this study was to investigate the causal relationship between smoking and depression/ anxiety, and specially the direction of this relationship, in order to analyze the link between the degree of dependence to nicotine, and the anxio-depressive levels, according to HADS test and the Fagerstrom test of nicotine dependence. Our study included 647 smokers and 400 non-smoking male. To facilitate this study, we distributed questionnaires to several private GP surgeries, to be completed by patients. In this study, we found that there is a close relationship between the level of nicotine addiction, anxiety levels and the emergence of depressive disorders. A highly depressive state implies a very strong addiction to tobacco; a high percentage of subjects showed little or no tobacco dependence. When anxiety appears (even when non-significant) dependence increases from medium to high, and very high, whereas in the absence of depression, $80 \%$ to $90 \%$ of patients show no tobacco addiction. The addiction to tobacco increases at the beginning of depression and reach her maximum in depressed patients. Thus, the anxiolytic effect of nicotine becomes an anxiogenic effect in anxious subjects, when subjects move from non-dependence to medium and very strong addiction.
\end{abstract}

Keywords: Nicotine; Tobacco; Addiction; Fagerstrom Test; HADS Test; Depression; Anxiety

\section{INTRODUCTION}

Smoking has many biological effects which contribute to its negative impact on health. These negative effects are well known and mortality caused by smoking is high [1]. Many studies have helped to understand the relationship between smoking and anxiety disorders [2-4]. Some

\footnotetext{
"Corresponding author.
}

studies have shown that people with mental disorders were twice as likely to smoke compared to the general population $[5,6]$ and are large consumers of tobacco [7]. According to Williams and Zidounis (2004) [8], 50\% to $90 \%$ of individuals suffering from mental disorders are highly dependent on tobacco. The degree of dependence varies with the pathologies and comorbidities. Overall, the literature shows that smoking patients are more depressed than nonsmokers [9], and nicotine dependence is greater in depressed people [10].

Smoking and anxiety are related, and some studies show an effect of smoking on anxiety, while others show an influence of anxiety on smoking. There is a relatively high rate of smokers among patients suffering of anxiety [3], and the presence of an anxiety disorder with or without depression is associated with a greater probability of smoking. Other studies indicate that smoking rates are higher in people having anxiety disorders compared to people who have no symptoms of depression and anxiety $[11,12]$. Anyway, the literature tells that smokers feel more anxious than nonsmokers $[12,13]$. On the other hand, the antidepressant properties of nicotine to reduce symptoms of depression [14,15] are a factor in maintaining smoking, because the smoker in need of nicotine feels depressed when he does not smoke. He begins to feel better when the effect of an antidepressant replaces the nicotine. Thus, administration of nicotine can cause psychological benefits like improving mood, concentration, vigilance and cognitive function [16,17]. Smokers use tobacco for self-medication to decrease the negative effects their illness or to improve some physiological functions [18]. We can therefore consider nicotine as a regulator of psychological balance [19].

Smoking has antidepressant effects because tobacco contains nicotine and other inhibitors of monoamine oxidase. These substances induce the releasing of chemical messengers and neurotransmitters, serotonin and norepinephrine, which play a role in the pleasure and comfort and can help the smoker in the management of 
his mood [20,21]. A link can be established between nicotine weaning and depressed mood. These painful sensations usually remain during ten to thirty days and rarely disappear after two months. Smoking is a risk factor for developing panic disorder, which is proportional to the degree of nicotine dependence [21]. Addiction is a psychological and sometimes physical state resulting from the interaction between a living organism and a foreign substance, characterized by behavioral responses with a compulsion to take continuously the substance or periodically to avoid psychic discomfort and sometimes to avoid the discomfort of its absence [19].

\section{OBJECTIVE OF THE STUDY}

The main objective of this study was to investigate the causal relationship between smoking and depression/ anxiety, and the direction of this relationship, in order to analyze the link between the degree of dependence to nicotine, and the anxio-depressive levels, according to HADS test and Fagerstrom test of nicotine dependence.

\section{SUBJECTS AND METHODS}

\subsection{Subject Assessment}

This study was approved by Scientific Council of the Faculty of Sciences, Annaba University, Algeria. A demographic questionnaire collected data about patient's characteristics (age, antecedent) and medical status, the Hospital Anxiety and Depression Scale (HADS) [22] and Fagerstrom Test for Nicotine Dependence [23] were used in this study (Figure 1). All subjects underwent a thorough medical evaluation including medical history, physical exam and laboratory exam (complete blood cell count, biochemical parameters). Subjects with clinically meaningful abnormal laboratory values were excluded.
Our study included 647 smokers and 400 non-smoking male. The survey was conducted from October 2011 to April 2012 in north eastern Algeria. To facilitate this study, we distributed questionnaires of Fagerstrom and HADS tests to several general practitioner offices, to be completed by anonymous smoking patients.

\subsection{Hospital Anxiety and Depression Scale (HADS)}

Comorbidity of anxiety and depression is estimated through the HADS test which contains fourteen items rated from 0 to 3 . Seven questions are related to anxiety (HADS A) and seven questions to depressive dimension (HADS D), in order to obtain two scores (maximum score $=21)$. By adding the points of the $1,3,5,7,9,11$, 13 responses, we obtain the A total, and for the $2,4,6,8$, $10,12,14$ answers, we obtain the $\mathrm{D}$ total. To detect depression or anxiety symptoms, an interpretation is proposed for each A and D scores. A score above 8 on the D scale indicates a depressive state and a score above 12 on the A scale indicates an anxiety disorder [24].

Patients are asked to choose one response from the four given for each interview. They should give an immediate response and be dissuaded from thinking too long about their answers. The questions relating to anxiety are marked "A", and to depression "D".

A I feel tense or "wound up":

Most of the time 3

A lot of the time 2

From time to time, occasionally 1

Not at all 0

D I still enjoy the things I used to enjoy:

Definitely as much 0

Not quite so much 1

Only a little 2

Hardly at all 3

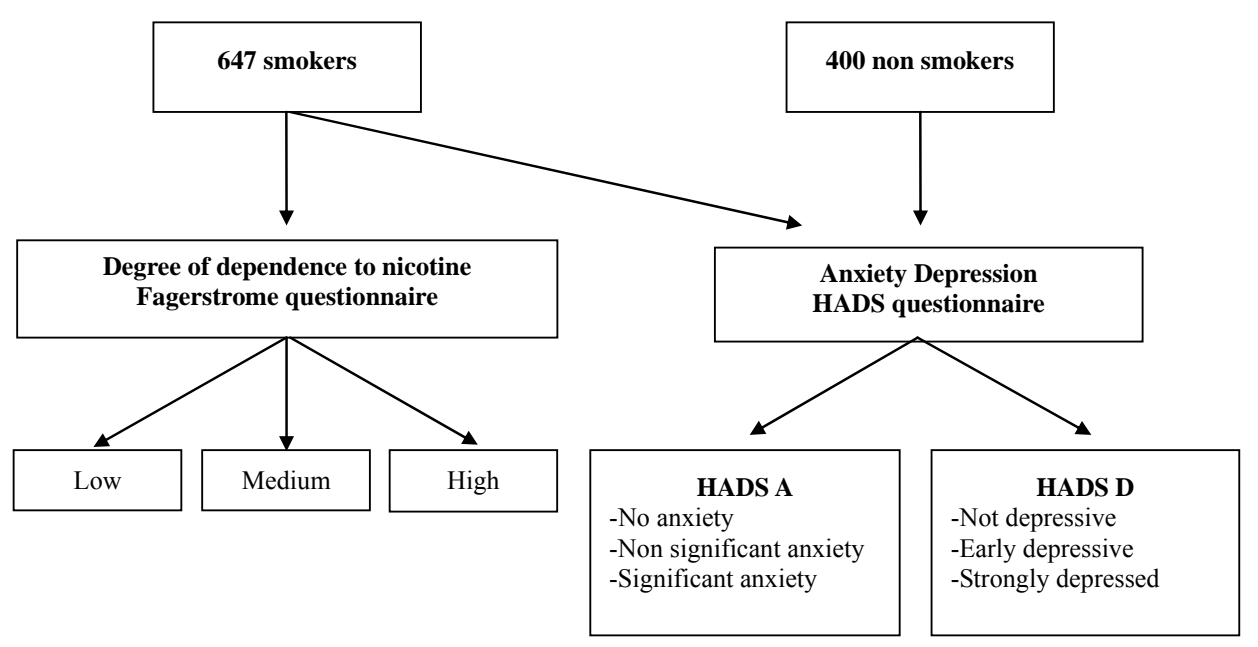

Figure 1. Experimental protocol. 
A I get a sort of frightened feeling as if something awful is about to happen:

Very definitely and quite badly 3

Yes, but not too badly 2

A little, but it doesn't worry me 1

Not at all 0

D I can laugh and see the funny side of things:

As much as I always could 0

Not quite so much now 1

Definitely not so much now 2

Not at all 3

A Worrying thoughts go through my mind:

A great deal of the time 3

A lot of the time 2

From time to time, but not too often 1

Only occasionally 0

D I feel cheerful:

Not at all 3

Not often 2

Sometimes 1

Most of the time 0

A I can sit at ease and feel relaxed:

Definitely 0

Usually 1

Not Often 2

Not at all 3

D I feel as if I am slowed down:

Nearly all the time 3

Very often 2

Sometimes 1

Not at all 0

A I get a sort of frightened feeling like "butterflies" in the stomach:

Not at all 0

Occasionally 1

Quite Often 2

Very Often 3

D I have lost interest in my appearance:

Definitely 3

I don't take as much care as I should 2

I may not take quite as much care 1

I take just as much care as ever 0

A I feel restless as I have to be on the move:

Very much indeed 3

Quite a lot 2

Not very much 1

Not at all 0

D I look forward with enjoyment to things:

As much as I ever did 0

Rather less than I used to 1

Definitely less than I used to 2

Hardly at all 3

A I get sudden feelings of panic:

Very often indeed 3
Quite often 2

Not very often 1

Not at all 0

D I can enjoy a good book or radio or TV program:

Often 0

Sometimes 1

Not often 2

Very seldom 3

Scoring (add the As $=$ Anxiety. Add the Ds $=$ Depression). The norms below will give an idea of the level of Anxiety and Depression.

$$
\begin{aligned}
& 0-7=\text { Normal } \\
& 8-10=\text { Borderline abnormal } \\
& 11-21=\text { Abnormal }
\end{aligned}
$$

\subsection{Fagerstrom Test for Nicotine Dependence}

The Fagerstrom test consists in six questions to measure the level of nicotine dependence. The total score is between 0 and 10, and the interpretation of test score is:

Score 0 to 2: the subject is not addicted to nicotine, it can often stop smoking without using nicotine replacement therapy.

Score of 3 to 4 : the subject is weakly dependent on nicotine.

Score of 5 to 6: the subject is moderately dependent on nicotine.

Score of 7 to 8: the subject is highly dependent on nicotine.

Score of 9 to 10 : the subject is very heavily dependent on nicotine [25].

\subsection{Questions of the Test}

Is smoking "just a habit" or are you addicted? Take this test and find out your level of dependence on nicotine.

1. How soon after you wake up do you smoke your first cigarette?

After 60 minutes (0)

$31-60$ minutes $(1)$

6 - 30 minutes (2)

Within 5 minutes (3)

2. Do you find it difficult to refrain from smoking in places where it is forbidden?

No (0)

Yes (1)

3. Which cigarette would you hate most to give up?

The first in the morning (1)

Any other $(0)$

4. How many cigarettes per day do you smoke?

10 or less $(0)$

$11-20(1)$

$21-30(2)$

31 or more (3)

5. Do you smoke more frequently during the first 
hours after awakening than during the rest of the day?

No $(0)$

Yes (1)

6. Do you smoke even if you are so ill that you are in bed most of the day?

No $(0)$

Yes (1)

Scores of the Fagerstrom Test for Nicotine Dependence

Your score was:

Your level of dependence on nicotine is:

0 - 2 Very low dependence

3 - 4 Low dependence

5 Medium dependence

6 - 7 High dependence

8 - 10 Very high dependence

Scores under 5: "Your level of nicotine dependence is still low. You should act now before your level of dependence increases."

Score of 5: "Your level of nicotine dependence is moderate. If you don't quit soon, your level of dependence on nicotine will increase until you may be seriously addicted. Act now to end your dependence on nicotine."

Score over 7: "Your level of dependence is high. You aren't in control of your smoking - it is in control of you! When you make the decision to quit, you may want to talk with your doctor about nicotine replacement therapy or other medications to help you break your addiction."

\section{RESULTS}

Fagerstrom and HADS test scores are presented in Table 1. The very high dependence to nicotine show 40 subjects $(6.18 \%)$, including 14 subjects $(35 \%)$ in significant anxiety state, $23(57.5 \%)$ in non-significant anxiety, and 03 non anxious $(7.5 \%)$.

About depressive disorders, 10 subjects $(25 \%)$ are in strong depressive state, 17 subjects $(42.5 \%)$ in early de- pression, and 13 are not depressive (32.5\%). Subjects who do not have anxiety show the lowest tobacco dependence, in non-dependence $(66.66 \%)$ and in very low dependence $(50.76 \%)$.

The strong dependence concerns 315 subjects (48.68\%), whom $72(22.85 \%)$ with significant anxiety state, 209 in non-significant anxiety state $(66.34 \%)$ and 34 non-anxious $(10.79 \%)$. Among this group, 6 persons $(1 \% 90)$ are highly depressive, $140(44.44 \%)$ in early depression and 169 are not depressive (53.65\%). In the non significant anxiety state, the highest percentage is medium, high and very strong tobacco dependence (respectively $56.79 \%$, $66.34 \%$ and $57.5 \%$ ).

In the medium dependence to tobacco, 206 subjects $(31.83 \%)$ have been recorded, including 40 subjects $(19.41 \%)$ in a significant state of anxiety, 117 subjects $(56.79 \%)$ in non-significant anxiety state, and 49 subjects $(23.78 \%)$ are not anxious. We noted 4 strongly depressed persons $(1.94 \%), 62(30.09 \%)$ in early depression and $140(67.96 \%)$ not depressive. It appears that when subjects are not depressed, they have just a small or no dependence on tobacco, with $80 \%$ and $90.48 \%$, implying an antidepressant effect of tobacco when consumed in small amounts.

In low nicotine dependence, our results show 65 subjects (10.04\%), including 2 individuals (3.07\%) in a significant state of anxiety, $30(46.15 \%)$ in a non significant state of anxiety, and 33 non-anxious $(50.76 \%)$. For depression, we observed no strong depressive state $(0 \%)$, 13 subjects (20\%) in early depression and no depression in $52(80 \%)$.

In early state of depression, the subjects showed increased levels of tobacco dependence when it is low (30.09\%), high (44.44\%) or extremely high $(42.5 \%)$.

Among the 21 subjects having no addiction to nicotine (3.24\%), there is no significant anxiety (0\%), $7(33.33 \%)$ in non-significant anxiety, and $14(66.66 \%)$ in no anxiety.

Table 1. Percent distribution of levels of anxiety and depression (HAD scale) depending on the degree of smoking addiction (Fagerstrom test) of 400 participants non-smokers and 647 smokers.

\begin{tabular}{|c|c|c|c|c|c|c|c|}
\hline \multirow{2}{*}{\multicolumn{2}{|c|}{ HAD scale }} & \multirow{2}{*}{$\begin{array}{c}\text { Control } \\
(\mathrm{n}=400)\end{array}$} & \multicolumn{5}{|c|}{ Smokers $(n=647)$ : Degree of dependence } \\
\hline & & & $\begin{array}{c}\text { No } \\
\text { dependence }\end{array}$ & $\begin{array}{c}\text { Weak } \\
\text { dependence }\end{array}$ & $\begin{array}{c}\text { Medium } \\
\text { dependence }\end{array}$ & $\begin{array}{c}\text { High } \\
\text { dependence }\end{array}$ & $\begin{array}{l}\text { Very high } \\
\text { dependence }\end{array}$ \\
\hline & Global repartition & $100 \%$ & $3.24 \%$ & $10.04 \%$ & $31.83 \%$ & $48.68 \%$ & $6.18 \%$ \\
\hline \multirow{3}{*}{$\begin{array}{c}\text { Anxiety } \\
\text { level }\end{array}$} & No anxiety & $58.75 \%$ & $66.66 \%$ & $50.76 \%$ & $23.78 \%$ & $10.79 \%$ & $7.5 \%$ \\
\hline & Non significant anxiety state & $24.25 \%$ & $33.33 \%$ & $46.15 \%$ & $56.79 \%$ & $66.34 \%$ & $57.5 \%$ \\
\hline & Significant anxiety state & $17 \%$ & $0 \%$ & $3.09 \%$ & $19.41 \%$ & $22.85 \%$ & $35 \%$ \\
\hline \multirow{3}{*}{$\begin{array}{l}\text { Degree of } \\
\text { depression }\end{array}$} & Not depressive & $90.25 \%$ & $90.48 \%$ & $80 \%$ & $67.96 \%$ & $53.65 \%$ & $32.5 \%$ \\
\hline & Early depression & $8.50 \%$ & $9.52 \%$ & $20 \%$ & $30.09 \%$ & $44.44 \%$ & $42.5 \%$ \\
\hline & Strongly depressed state & $1.25 \%$ & $0 \%$ & $0 \%$ & $1.94 \%$ & $1.90 \%$ & $25 \%$ \\
\hline
\end{tabular}


There is no subject in strong depressive state, $2(9.52 \%)$ in early depression, and 19 (90.48\%) had no depression.

When subjects are strongly depressed, 25\% have a very high tobacco dependence. In a significant state of anxiety, there are no issues involved $(0 \%)$ both in the small or no dependence to tobacco. From medium $(19.41 \%)$, high $(22.85 \%)$ or extremely high dependence $(35 \%)$, subjects move from anxiolytic to anxiogenic effect of nicotine.

In the 400 non-smoking participants (controls) 235 subjects $(58.75 \%)$ were in a normal state. The anxiety was non significant for 97 subjects $(24.25 \%)$, significant for 68 subjects (17), 90.25\% showing an absence of depressive disorders, $8.5 \%$ early depressive and $1.25 \%$ strongly depressive .

\section{DISCUSSION}

In spite of many studies on neurological effects of tobacco, it has not been clear whether anxiety, depressive disorders and mood disturbances are more frequent in patients addicted to nicotine compared to less dependent or non-smokers [26,27].

In this study, we found that there is a close relationship between the level of nicotine addiction, anxiety levels and the emergence of depressive disorders. We also noted that nicotine has an anxiolytic effect in most levels of nicotine dependence, and these results confirm the anxiolytic effect of nicotine reported in previous research. The test most frequently used to measure the anxiolytic effects of a substance is the Elevated Plus Maze, and a sub acute or chronic injection of nicotine increases the time spent by rats in the open arms. According to some authors, the anxiolytic effects of nicotine have a peripheral origin [28], while for others, serotoninergic neurons of the raphe nucleus are essential because the anxiolytic effect of nicotine is inhibited by serotonin antagonists in the dorsal raphe nucleus, which produces an anxiolytic effect similar to that obtained by peripheral injection of nicotine [29].

Our results show a bidirectional effect of tobacco: an anxiolytic effect, probably through its inclusion in the behavioral repertoire of stress management of smoking, and an anxiogenic effect in smokers highly dependent on nicotine [30]. We can also observe that the anxiolytic effect of nicotine is effective only among subjects without anxiety and characterized by little or no dependence on tobacco. By cons, anxiogénique effect of nicotine has been proved when the subject reach a low but real state of anxiety (not significant), since the degree of dependence to nicotine becomes medium, strong and very strong. Thus the passage from an anxiolytic effect of nicotine to an antidepressant effect marks the beginning of a depression in patients who begin to show a medium, then strong and very strong dependence on nicotine. In strongly depressed persons, subjects have a very strong degree of dependence on tobacco. This can be seen in patients undergoing the test of the Hamilton depression (HAM-D) showing a significant increase in improvement in depression after the second day of using nicotine patches, but relapsed 3 or 4 days after the last dose of nicotine, indicating that patches produce a short-term improvement of depression with minimal side effects [31].

The administration of nicotine can cause a psychological benefit such as improved mood, concentration, alertness and cognitive functioning [16,17]. From these observations, a self-medication hypothesis could be considered, that smoking may alleviate some symptoms of depression through the actions of nicotine, the first component psycho active of smoking [32,33]. Another behavioral study in rodents showed that chronic administration of nicotine has antidepressant effects through its actions on specific subtypes of nicotinic acetylcholine receptors [34-36]. It has moreover been shown that chronic administration of low doses of nicotine in humans is clinically effective as an antidepressant [37,38]. Tobacco smoke is a potent inhibitor of monoamine oxidase (MAO), whereas MAO plays an important role in some psychiatric behaviors such as depression $[39,40]$. The inhibition of MAO results in increased brain levels of biogenic amines, including norepinephrine and serotonin, which are usually reduced in depression pathology [41]. A previous study suggested that levels of monoamine oxidase A enzyme (MAO A) and MAO B, are reduced in platelets and brain of smokers. The inhibition of MAO activity by compounds present in tobacco may be combined with nicotine to strongly enhance the action of smoking, leading to dependence [42]. This effect was proposed to be involved in the antidepressant properties of tobacco [20,21], and many studies have shown an association between smoking and depression [21], the development of dependence to nicotine being clearly associated with mental disorders $[42,43]$. Some studies showed that the number of anxious smokers was higher than nonsmokers $[12,44]$ and smoking could be a comorbidity with anxiety [45]. There is also a significant comorbidity of anxiety disorders and mood, and up to $90 \%$ of patients suffering of anxiety had also a depression [46].

In our study, we suggested that the anxiogenic effect of nicotine appears in subjects heavily dependent on tobacco, compared to other levels of dependency, and the same results were obtained by Ziedonis et al. (2008) [47], whereas the association is stronger between high anxiety and nicotine dependence, compared with less dependent smokers and non smokers. There is also evidence of a strong association between common mental disorders 
and nicotine dependence $[7,48]$. That mean that smoking would have an effect on the onset of depression, as suggested in a longitudinal study over a period of 26 years and confirm this trend. Indeed, smokers without depression were more likely to develop depression following their smoking compared to non smokers. Smoking appears to be a risk factor for depression and it would exist a dose-response effect: the higher the amount of tobacco consumed is large, and more the rate of depression is high, [49]. Thus, tobacco use, translated in level of anxiety, in degree of depression and in addiction, allows to establish a human model of addiction to smoking and may be a predictor of depression, besides the other means of investigation.

\section{CONCLUSIONS}

It appears in this study, conducted on a sample of 647 smokers and 400 non-smokers (controls), that a highly depressive state implies a very strong addiction to tobacco. Tests of Fagrestrome and HADS helped to define the degree of tobacco dependence according to the state of anxiety and depression, and showed that in lack of anxiety, a high percentage of subjects showed small or no tobacco dependence. When anxiety appears (even when it is non-significant) dependence increases from medium to high, and very high, whereas in absence of depression, $80 \%$ to $90 \%$ of patients show no tobacco addiction. The dependence increases at the beginning of depression and reach her maximum in depressed patients. Thus, the anxiolytic effect of nicotine becomes an anxiogenic effect in anxious subjects, when transition occurs from non-dependence to a medium dependency.

Nicotine is known to be antidepressant, and the in need subject feels depressed. He begins to smoke by self-medication, and it helps him to manage his mood. This bidirectional effect between smoking, anxiety/depression and anxio-depression levels, raise the problem of the direction of the influence of these factors: smoking affects anxiety and depression, depression make the subject smoking more and more.

\section{ACKNOWLEDGEMENTS}

We gratefully acknowledge Dr. BOUDJNIBA Mahaiedine, Dr. BOUTERRENE Aziz and Dr. DJARRAB Hassene for their contribution and support throughout this study.

\section{REFERENCES}

[1] Doll, R., Peto, R., Boreham, J. and Sutherland, I. (2004) Mortality in relation to smoking: 50 years' observations on male British doctors. British Medical Journal, 328, 1519-1528. doi:10.1136/bmj.38142.554479.AE

[2] Brown, P.J. and Wolfe, J. (1994) Substance abuse and post-traumatic stress disorder co-morbidity. Drug and Alcohol Dependence, 35, 51-59.

doi:10.1016/0376-8716(94)90110-4

[3] Patton, G.C., Carlin, J.B., Coffey, C., Wolfe, R., Hibbert, M. and Bowes, G. (1998) Depression, anxiety, and smoking initiation: A prospective study over 3 years. American Journal of Public Health, 88, 1518-1522. doi:10.2105/AJPH.88.10.1518

[4] Kalman, D., Morissette, S.B. and George, T.P. (2005) Co-morbidity of smoking in patients with psychiatric and substance use disorders. The American Journal of Addictions, 14, 106-123. doi:10.1080/10550490590924728

[5] Lasser, K., Boyd, J.W., Woolhandler, S., Himmelstein, D.U., McCormick, D. and Bor, D.H. (2000) Smoking and mental illness: A population-based prevalence study. Journal of the American Medical Association, 284, 26062610. doi:10.1001/jama.284.20.2606

[6] Olivier, D., Lubman, D.I. and Fraser, R. (2007) Tobacco smoking within psychiatric inpatient settings: A biopsycho social perspective. Australian and New Zealand. Journal of Psychiatry, 41, 572-580. doi:10.1080/00048670701392809

[7] Grant, B.F., Hasin, D.S., Chou, S.P., Stinson, F.S. and Dawson, D.A. (2004) Nicotine dependence and psychiatric disorders in the United States. Results from the national epidemiologic survey on alcohol and related conditions. Archives of General Psychiatry, 61, 1107-1115. doi:10.1001/archpsyc.61.11.1107

[8] Williams, J.M. and Ziedonis, D. (2004) Addressing tobacco among individuals with mental illness or an addiction. Addictive Behaviour, 29, 1067-1083. doi:10.1016/j.addbeh.2004.03.009

[9] Glasman, A.H., Covey, L.S., Stentner, F. and Revelli, S. (2001) Smoking cessation and the course of major depression: A follow-up study. Lancet, 357, 1929-1932.

[10] Glasman, A.H., Helzer, J.E., Covey, L.S., Cottler, L.B., Stentner, F., Tipp, J.E. and Johnson, J. (1990) Smoking, smoking cessation, and major depression. Journal of the American Medical Association, 264, 1546-1554. doi:10.1001/jama.1990.03450120058029

[11] Goodwin, R.D., Zvolensky, M.J. and Keyes, K.M. (2008) Nicotine dependence and mental disorders among adults in the USA: Evaluating the role of the mode of administration. Psychological Medicine, 8, 1277-1286. doi:10.1017/S0033291708003012

[12] Mc Cabe, R.E., Chudzik, S.M., Antony, M.M., Young, L., Swinson, R.P. and Zolvensky, M.J. (2004) Smoking behaviours across anxiety disorders. Journal of Anxiety Disorders, 18, 7-8. doi:10.1016/j.janxdis.2003.07.003

[13] Morissette, S.B., Brown, T.A., Kamholz, B.W. and Gulliver, S.B. (2006) Difference between smokers and nonsmokers with anxiety disorders. Journal of Anxiety Disorders, 20, 597-613. doi:10.1016/j.janxdis.2005.08.003

[14] Audrain-McGovern, J., Rodriguez, D. and Kassel, J.D. (2009) Adolescent smoking and depression: Evidence for self-medication and peer smoking mediation. Addiction, 104, 1743-1756. doi:10.1111/j.1360-0443.2009.02617.x

[15] Paperwalla, K.N., Levin, T.T., Weiner, J. and Saravay, 
S.M. (2004) Smoking and depression. The Medical Clinics of North America, 88, 1483-1494.

doi:10.1016/j.mcna.2004.06.007

[16] Benowitz, N.L. (2008) Clinical pharmacology of nicotine: Implications for understanding, preventing, and treating tobacco addiction. Clinical Pharmacology and Therapeutics, 83, 531-541. doi:10.1038/clpt.2008.3

[17] Parrott, A.C. (2006) Nicotine psychobiology: How chronic-dose prospective studies can illuminate some of the theoretical issues from acute-dose research. Psychopharmacology, 184, 567-576. doi:10.1007/s00213-005-0294-y

[18] Chaiton, M., Cohen, J., O'Loughlin, J. and Rehm, J. (2010) Use of cigarettes to improve affect and depressive symptoms in a longitudinal study of adolescents. Addictive Behaviour, 35, 1054-1060. doi:10.1016/j.addbeh.2010.07.002

[19] Lagrue, G. (2004) Traitement de la dépendance tabagique. Centre de tabacologie, Hôpital Albert Chenevier, Créteil.

[20] Fowler, J.S., Volkow, N.D., Wang, G.J., Pappas, N., Logan, J., Shea, C., Alexoff, D., MacGregor, R., Schlyer, D.J., Zezulkova, I. and Wolf, A.P. (1996) Brain monoaminooxidase A inhibition in cigarette smokers. Proceedings of the National Academy of Sciences USA, 93, 14065-14069. doi:10.1073/pnas.93.24.14065

[21] Fowler, J.S., Volkow, N.D., Wang, G.J., Pappas, N., Logan, J., MacGregor, R., Alexoff, D., Wolf, A.P., Warner, D., Cilento, R. and Zezulkova, I. (1998) Neuropharmacological actions of cigarette smoke: Brain monoaminooxidase B (MAO B) inhibition. Journal of Addictive Diseases, 17, 23. doi:10.1300/J069v17n01_03

[22] Zigmond, A.S. and Snaith, R.P. (1983) The hospital anxiety and depression scale. Acta Psychiatrica Scandinavica, 67, 361-370. doi:10.1111/j.1600-0447.1983.tb09716.x

[23] Fagerstrom, K.O., Heatherton, T. and Kozlowski, L.T. (1991) Nicotine addiction and its assessment. Ear, Nose and Throat Journal, 69, 763-766.

[24] Zigmond, A.S. and Snaith, R.P. (1983) The hospital anxiety and depression scale. Acta Psychiatrica Scandinavica, 67, 361-370.

doi:10.1111/j.1600-0447.1983.tb09716.x

[25] Etter, J.F., Duc, T.V. and Pemeger, T.V. (1999) Validity of the Fagerstrom test for nicotine dependence and of the heaviness of smoking index among relatively light smokers. Addiction, 94, 269-281. doi:10.1046/j.1360-0443.1999.94226910.x

[26] Weinberger, A.H., Desai, R.A. and McKee, S.A. (2010) Nicotine withdrawal in US smokers with current mood, anxiety, alcohol use, and substance use disorders. Drug and Alcohol Dependence, 108, 7-12. doi:10.1016/j.drugalcdep.2009.11.004

[27] Ziedonis, D., Hitsman, B., Beckham, J.C., Zvolensky, M., Adler, L.E., Audrain-McCovern, J., et al. (2008) Tobacco use and cessation in psychiatric disorders: National Institute of Mental Health report. Nicotine \& Tobacco Research, 10, 1691-1715.

doi: $10.1080 / 14622200802443569$
[28] Ericson, M., Olausson, P., Enrgl, J.A. and Soderpal, M.B. (2000) Nicotine induces disinhibitory behavior in the rat after subchronic peripheral nicotinic acetylcholine receptor blockade. European Journal of Pharmacology, 397, 103-111. doi:10.1016/S0014-2999(00)00191-6

[29] Chetta, S., Irvine, E.E., Kenny, P.J. and File, S.E. (2001) The dorsal raphe nucleus is a crucial structure mediating nicotine's anxiolytic effects and the development of tolerance and withdrawal responses. Psychopharmacology, 155, 78-85.

[30] Khazaal, Y., Cornuz, J. and Zullino, D. (2004) Les troubles anxieux sont-ils associés à un tabagisme particulier? Stress-anxiété et tabagisme. Santé Mentale au Québec, 29, 73-80. doi:10.7202/008821ar

[31] Salin-Pascual, R.J., Rosas, M., Jimenez-Genchi, A., Rivera-Meza, B.L. and Delgado-Parra, V. (1996) Antidepressant effect of transdermal nicotine patches in nonsmoking patients with major depression. Journal of Clinical Psychiatry, 57, 387-389.

[32] Markou, A., Kosten, T.R. and Koob, G.F. (1998) Neurobiological similarities in depression and drug dependence: A self-medication hypothesis. Neuropsychopharmacology, 18, 135-174. doi:10.1016/S0893-133X(97)00113-9

[33] Tizabi, Y., Overstreet, D.H., Rezvani, A.H., Louis, V.A., Clark, E. Jr., Janowsky, D.S. and Kling, M.A. (1999) Antidepressant effects of nicotine in an animal model of depression. Psychopharmacology, 142, 193-199. doi:10.1007/s002130050879

[34] Buckley, M.J., Surowy, C., Meyer, M. and Curzon, P. (2004) Mechanism of action of A-85380 in an animal model of depression. Progress in Neuro-Psychopharmacology \& Biological Psychiatryis, 28, 723-730. doi:10.1016/j.pnpbp.2004.05.012

[35] Ferguson, S.M., Brodkin, J.D., Lloyd, G.K. and Menzaghi, F. (2000) Antidepressant-like effects of the subtype-selective nicotinic acetylcholine receptor agonist, SIB-1508Y, in the learned helplessness rat model of depression. Psychopharmacology, 152, 295-303. doi:10.1007/s002130000531

[36] Semba, J., Mataki, C., Yamada, S., Nankai, M. and Toru, M. (1998) Antidepressant-like effects of chronic nicotine on learned helplessness paradigm in rats. Biological Psychiatry, 43, 389-391. doi:10.1016/S0006-3223(97)00477-0

[37] Van Riezen, H. and Leonard, B.E. (1990) Effects of psychotropic drugs on the behaviour and neurochemistry of olfactory bulbectomized rats. Pharmacology \& Therapeutics, 47, 21-34. doi:10.1016/0163-7258(90)90043-2

[38] Cryan, J.F. and Mombereau, C. (2004) In search of a depressed mouse: Utility of models for studying depression-related behaviour in genetically modified mice. Molecular Psychiatry, 9, 326-357. doi:10.1038/sj.mp.4001457

[39] Mc Daniel, K. (1986) Clinical pharmacology of monoamine oxidase inhibitors. Clinical Neuropharmacology, 9, 207-234. doi:10.1097/00002826-198606000-00001

[40] Yamada, M. and Yasuhara, H. (2004) Clinical pharmacology of MAO inhibitors: Safety and future. Neurotoxi- 
cology, 25, 215-221. doi:10.1016/S0161-813X(03)00097-4

[41] Herraiz, T. and Chaparro, C. (2005) Human monoamine oxidase is inhibited by tobacco smoke: $\beta$-carboline alkaloids act as potent and reversible inhibitors. Biochemical and Biophysical Research Communications, 326, 378386. doi:10.1016/j.bbrc.2004.11.033

[42] Dierker, L. and Donny, E. (2008) The role of psychiatric disorders in the relationship between cigarette smoking and DSM-IV nicotine dependence among young adults. Nicotine \& Tobacco Research, 10, 439-446. doi:10.1080/14622200801901898

[43] Goodwin, R.D., Zvolensky, M.J. and Keyes, K.M. (2008) Nicotine dependence and mental disorders among adults in the USA: Evaluating the role of the mode of administration. Psychological Medicine, 38, 1277-1286. doi:10.1017/S0033291708003012

[44] Strine, T.W., Mokdad, A.H., Balluz, L.S., Berry, J.T. and Gonzalez, O. (2008) Impact of depression and anxiety on quality of life, health behaviours, and asthma control among adults in the United States with asthma. Journal of Asthma, 45, 123-133. doi:10.1080/02770900701840238
[45] Morrell, H.E.R. and Cohen, L.M. (2006) Cigarette smoking, anxiety, and depression. Journal of Psychopathology and Behavioural Assessment, 28, 281-295. doi:10.1007/s10862-005-9011-8

[46] Gorman, J.M. (1996) Comorbid depression and anxiety spectrum disorders. Depression and Anxiety, 4, 160-168. doi:10.1002/(SICI)1520-6394(1996)4:4<160::AID-DA2> 3.0.CO;2-J

[47] Ziedonis, D., Hitsman, B., Beckham, J.C., Zvolensky, M., Adler, L.E., Audrain-McCovern, J., et al. (2008) Tobacco use and cessation in psychiatric disorders: National Institute of Mental Health report. Nicotine \& Tobacco Research, 10, 1691-1715. doi:10.1080/14622200802443569

[48] Breslau, N., Novak, S.P. and Kessler, R.C. (2004) Psychiatric disorders and stages of smoking. Biological Psychiatry, 55, 69-76. doi:10.1016/S0006-3223(03)00317-2

[49] Flensborg-Madsen, T., Von Scholten, M.B., Flachs, E.M., Mortensen, E.L., Prescott, E. and Tolstrup, J.S. (2011) Tobacco smoking as a risk factor for depression. A 26year population-based follow-up study. Journal of Psychiatric Research, 45, 143-149. doi:10.1016/j.jpsychires.2010.06.006 